\title{
Magnetostriction and Magnetic Heterogeneities in Iron-Gallium
}

\author{
M. Laver, ${ }^{1,2,3,4}$ C. Mudivarthi, ${ }^{4}$ J. R. Cullen, ${ }^{4}$ A. B. Flatau, ${ }^{4,5}$ W.-C. Chen, ${ }^{6,7}$ S. M. Watson, ${ }^{6}$ and M. Wuttig ${ }^{4}$ \\ ${ }^{1}$ Laboratory for Neutron Scattering, Paul Scherrer Institut, CH 5232 Villigen, Switzerland \\ ${ }^{2}$ Materials Research Division, Risф DTU, Technical University of Denmark, DK-4000 Roskilde, Denmark \\ ${ }^{3}$ Nano-Science Center, Niels Bohr Institute, University of Copenhagen, DK-2100 Kфbenhavn, Denmark \\ ${ }^{4}$ Department of Materials Science and Engineering, University of Maryland, College Park, Maryland 20742, USA \\ ${ }^{5}$ Department of Aerospace Engineering, University of Maryland, College Park, Maryland 20742, USA \\ ${ }^{6}$ NIST Center for Neutron Research (NCNR), Gaithersburg, Maryland 20899, USA \\ ${ }^{7}$ Indiana University, Bloomington, Indiana 47408, USA \\ (Received 29 January 2010; published 8 July 2010)
}

\begin{abstract}
Iron-gallium alloys $\mathrm{Fe}_{1-x} \mathrm{Ga}_{x}$ exhibit an exceptional increase in magnetostriction with gallium content. We present small-angle neutron scattering investigations on a $\mathrm{Fe}_{0.81} \mathrm{Ga}_{0.19}$ single crystal. We uncover heterogeneities with an average spacing of $15 \mathrm{~nm}$ and with magnetizations distinct from the matrix. The moments in and around the heterogeneities are observed to reorient with an applied magnetic field or mechanical strain. We discuss the possible roles played by nanoscale magnetic heterogeneities in the mechanism for magnetostriction in this material.
\end{abstract}

PACS numbers: 75.80.+q, 61.05.fg, 61.72.Dd, 75.50.Bb

Pure iron exhibits only a small magnetostriction, which may be quantified by measuring the strain $\lambda_{\mathbf{p}}$ in a particular direction $\mathbf{p}$ when an initially demagnetized sample is magnetized to saturation. For pure bcc iron, $\lambda_{100}=20 \mathrm{ppm}$ and $\lambda_{111}=-16 \mathrm{ppm}$ at room temperature [1]. Surprisingly, adding nonmagnetic $\mathrm{Ga}$ to form $\mathrm{Fe}_{1-x} \mathrm{Ga}_{x}$ (galfenol) yields magnetostriction $\lambda_{100}>250 \mathrm{ppm}$ for quenched single crystals, with maxima occurring at $x \simeq$ 0.20 and $x \simeq 0.29$ [2]. With low hysteresis and favorable mechanical properties [3], iron-gallium alloys become auspicious for prominent applications ranging from artificial cilia transducers to microactuators $[4,5]$.

The mechanism behind the remarkable magnetostriction in $\mathrm{Fe}-\mathrm{Ga}$ has prompted much recent controversy [6-8]. In pure $\mathrm{Fe}$ the shape change is generated first by spatial shifts of magnetic domain walls and then by coherent rotation of the magnetization away from the easy direction in a process called Joule magnetostriction [9]. By contrast, in one of the proposed mechanisms for $\mathrm{Fe}-\mathrm{Ga}$, nanoscale heterogeneities generate strain by corporeal reorientation of tetragonal $\mathrm{DO}_{22}$ minidomains making up each heterogeneity [7]. Cubic heterogeneities are readily observed for $x \gtrsim 0.15$ [10]. There is one report of tetragonal heterogeneities [11], but no evidence yet links heterogeneities to magnetostriction.

In this Letter we report on small-angle neutron scattering (SANS) measurements using both polarized and unpolarized neutrons, performed at the NG3-SANS and NG7-SANS instruments at the NCNR (Gaithersburg, MD). SANS is a powerful technique [12] since not only can the bulk of a material be explored, but the magnetic nature of nanostructures is revealed through the particular character of the interaction between neutron spin and atomic moments, resulting in scattering patterns of distinct anisotropies on the 2D SANS detector, as parametrized by the angle $\theta$ of the scattering vector $\mathbf{q}$ in the detector plane [Fig. 1(a)]. Figure 1 shows the result of applying a large magnetic field $(\geq 1.3 \mathrm{~T})$ to our $\mathrm{Fe}_{0.81} \mathrm{Ga}_{0.19}$ sample. A $\sin ^{2} \theta \cos ^{2} \theta$ anisotropy is observed for polarized neutrons that scatter and flip their spin [Fig. 1(a)], a $\sin ^{4} \theta$ anisotropy for polarized neutrons that scatter without spin flip [Fig. 1(b)], and $\sin ^{2} \theta$ anisotropy for unpolarized neutrons
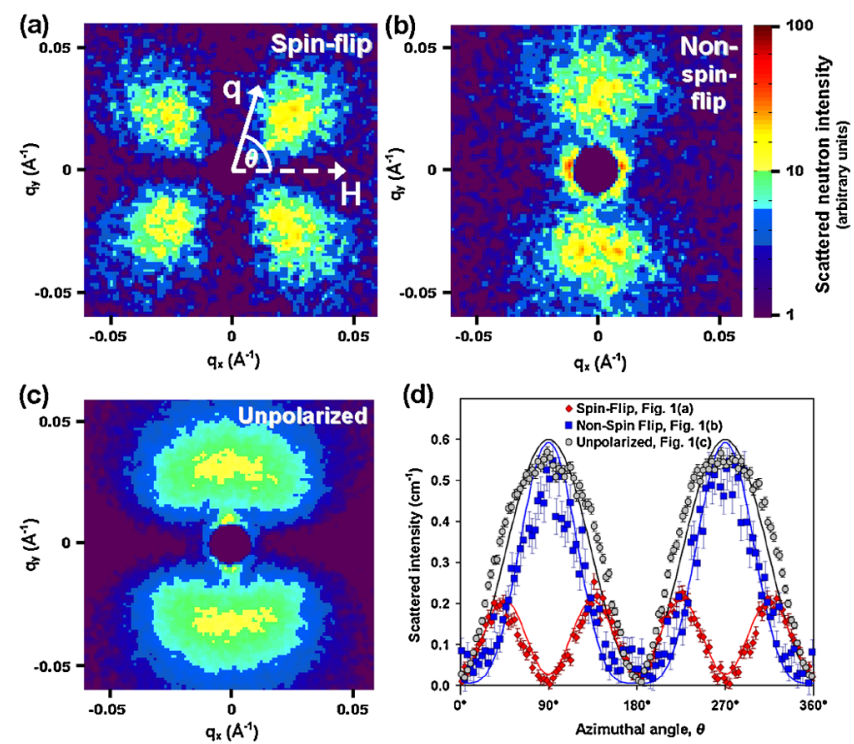

FIG. 1 (color online). 2D SANS detector images from our $\mathrm{Fe}_{0.81} \mathrm{Ga}_{0.19}$ single crystal at high fields $\geq 1.3 \mathrm{~T}$, using (a) polarized neutrons that scatter with spin flip or (b) without spin flip; (c) unpolarized neutrons. The incident beams along $\hat{\mathbf{z}}$ pass through the image centers, which have been masked. (d) $\theta$ dependence of the scattering, summed over $0.025<q<$ $0.05 \AA^{-1}$. Solid lines are fits described in the text. 
[Fig. 1(c)]. We will show that these patterns arise due to nanoscale heterogeneities whose magnetizations are distinct from the surrounding matrix.

Our $25 \times 17 \times 0.63 \mathrm{~mm}^{3} \quad \mathrm{Fe}_{0.81} \mathrm{Ga}_{0.19}$ single crystal was cut from a boule grown by the Bridgeman technique at a growth rate of $6 \times 10^{-7} \mathrm{~ms}^{-1}$, and subsequently quenched from $850^{\circ} \mathrm{C}$ to room temperature. Quenching boosts the magnetostriction [13] while suppressing the formation of ordered bcc $\left(\mathrm{DO}_{3}\right)$ structures in the chemically disordered bcc (A2) matrix [10]. The sample was then irradiated at $100{ }^{\circ} \mathrm{C}$ with a $0.1 \mathrm{~A}$ flux of $3 \mathrm{MeV}$ electrons for $1.7 \mathrm{~h}$ with the aim of increasing the formation of possible magnetostriction-generating heterogeneities. We have also observed in nonirradiated crystals of $0.15 \lessgtr x \leqq$ 0.20 heterogeneities on the nanoscale, similar to those detailed here for $x=0.19$. Our SANS studies were performed using cold neutrons of mean wavelengths between 5 and $7 \AA$, with $15 \%$ FWHM wavelength spread. In our polarized experiments, incident neutrons were polarized using a $\mathrm{Fe} / \mathrm{Si}$ transmission polarizer, and final neutron states were analyzed using a cell of nuclear-spin-polarized ${ }^{3} \mathrm{He}$. Starting with the results of Ref. [14], we obtain for both polarized and unpolarized neutrons intuitive forms for the differential cross sections $d \sigma / d \Omega$ from a volume $V$ of scatterers with moments $\mathbf{M}_{j}$ placed at $\mathbf{R}_{j}$, of nuclear and magnetic form factors $n_{j}(\mathbf{q}), m_{j}(\mathbf{q})$, respectively. We find it useful to work in cylindrical coordinates $(r, \theta, z)$ where $\hat{\mathbf{z}}$ is the beam direction. Then in the small-angle limit $\mathbf{q}=$ $(q, \theta, 0)$, and the component of $\mathbf{M}_{j}=\left(C_{j}, \phi_{j}, Z_{j}\right)$ in the $(r, \theta, 0)$ detector plane is explicit. For the polarized case, we consider incident neutrons polarized perpendicular to the beam direction, without loss of generality along $\theta=0$. Then

$$
\begin{aligned}
& \frac{d \sigma}{d \Omega}(\mathbf{q})=\frac{1}{V} \sum_{j, l} e^{-i \mathbf{q} \cdot\left(\mathbf{R}_{j}-\mathbf{R}_{l}\right)}\left\{n_{j}(\mathbf{q}) n_{l}^{*}(\mathbf{q})\right. \\
& +m_{j}(\mathbf{q}) m_{l}^{*}(\mathbf{q})\left[Z_{j} Z_{l}+C_{j} C_{l} \sin \left(\theta-\phi_{j}\right)\right. \\
& \left.\left.\times \sin \left(\theta-\phi_{l}\right)\right]\right\} \text {, } \\
& \frac{d \sigma^{ \pm \mp}}{d \Omega}(\mathbf{q})=\frac{1}{V} \sum_{j, l} e^{-i \mathbf{q} \cdot\left(\mathbf{R}_{j}-\mathbf{R}_{l}\right)} m_{j}(\mathbf{q}) \\
& \times m_{l}^{*}(\mathbf{q})\left[Z_{j} Z_{l}+C_{j} C_{l} \sin \left(\theta-\phi_{j}\right)\right. \\
& \left.\times \sin \left(\theta-\phi_{l}\right) \cos ^{2} \theta\right] \text {, } \\
& \frac{d \sigma^{ \pm \pm}}{d \Omega}(\mathbf{q})=\frac{1}{V} \sum_{j, l} e^{-i \mathbf{q} \cdot\left(\mathbf{R}_{j}-\mathbf{R}_{l}\right)}\left\{n_{j}(\mathbf{q}) n_{l}^{*}(\mathbf{q})\right. \\
& \mp\left[m_{j}(\mathbf{q}) n_{l}^{*}(\mathbf{q}) C_{j} \sin \left(\theta-\phi_{j}\right)\right. \\
& \left.+n_{j}(\mathbf{q}) m_{l}^{*}(\mathbf{q}) C_{l} \sin \left(\theta-\phi_{l}\right)\right] \\
& \times \sin \theta+m_{j}(\mathbf{q}) m_{l}^{*}(\mathbf{q}) C_{j} C_{l} \sin \left(\theta-\phi_{j}\right) \\
& \left.\times \sin \left(\theta-\phi_{l}\right) \sin ^{2} \theta\right\} \text {. }
\end{aligned}
$$

Equation (1a) describes the unpolarized case. Equations (1b) and (1c), describe polarized experiments where the final state is analyzed and the neutron scattered either with a spin flip or without spin flip, respectively. In Eq. (1b) we have omitted terms arising from helical spin structures [14]. For SANS, it is convenient to treat scattering variables as coarse grained rather than examining individual nuclei or atomic moments. Form factors $n, m$ are assessed by "scattering length density" (SLD), and M becomes a coarse-grained magnetization $\mathbf{M}(\mathbf{R})$. Further, scattering requires contrast in these quantities with respect to the matrix [12].

Our sample was mounted in an electromagnet providing fields in the horizontal detector direction $\hat{\mathbf{x}}$. Neutron spins reorient to track local fields providing their Larmor precession is fast enough or the field gradient is small enough [15]. For cold neutrons this adiabaticity limit $\approx 1 \mathrm{~T} /$ $\mu \mathrm{m} \gg$ gradient of electromagnet stray field. Therefore in our polarized experiments at high fields (Fig. 1), the neutron polarization, i.e., $\theta=0$ direction in Eqs. (1b) and (1c), is set along $\hat{\mathbf{x}}$. At lower fields below saturation, sample domains of differing magnetizations will similarly depolarize an initially polarized beam depending upon the domain size [16].

Our $\mathrm{Fe}_{0.81} \mathrm{Ga}_{0.19}$ crystal's $\langle 100\rangle$ axes were aligned with the beam direction $\hat{\mathbf{z}}$ and the horizontal and vertical detector directions $\hat{\mathbf{x}}$ and $\hat{\mathbf{y}}$. All measurements were performed at room temperature. Backgrounds, measured without the sample in the beam, were subtracted. The strains arising from applied magnetic fields were determined using electrical-resistance foil strain gauges affixed to the sample surface. For measurements under applied strain, an Al mount was used to apply uniaxial compression along $\hat{\mathbf{y}}$ while the gauges monitored the strain.

Comparing Eqs. (1) and the $\theta$ dependencies of the data at high magnetic fields [Fig. 1(d)], we see $\phi(\mathbf{R})=0$ everywhere, i.e., all moments are aligned parallel to the applied field, as expected at saturation. The magnetic scattering $m(\mathbf{q})|\mathbf{M}|=m(\mathbf{q}) C$ is clearly much greater than the nuclear component $n(\mathbf{q})$. We emphasize that the magnitude of the heterogeneity magnetization differs from that of the matrix. In Fig. 2 we plot the $q$ dependence at selected magnetic fields. At low $q$ the scattering at low fields is several orders of magnitude stronger than at high fields, and arises from ferromagnetic (FM) domains on scales $D$ permeating through the sample. This low $q$ scattering fits a power law $q^{-d}$ with $d \simeq 4.1$, characteristic of a Porod regime where $q D \gg 1$ [17]. From Fig. 2, $D \gg$ $300 \AA$, consistent with micrometer FM domains imaged by microscopy [18]. A weak power law in the scattering remains at saturating fields, likely due to crystal grain boundaries $[19,20]$.

The peak at $q=0.034 \AA^{-1}$ in Fig. 2 engenders the anisotropic 2D patterns of Fig. 1, but appears independent of field when summed over $\theta$ as in Fig. 2. We associate this prominent peak with the spatial distribution of heterogeneities, and accordingly model the heterogeneity scattering with a stochastic phenomenological structure factor [21] combined with a spheroidal form factor. A similar model was used to describe scattering from nanoprecipitates in a 


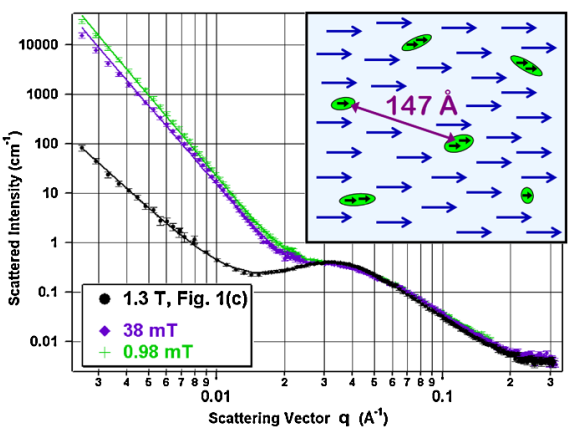

FIG. 2 (color online). $q$ dependence (summing over all $\theta$ ) of unpolarized neutron scattering at selected applied magnetic fields. Solid lines are fits using the scattering model described in the text. The inset is a schematic illustration of the local magnetizations at saturating fields.

nickel alloy [20] where ellipsoidal precipitates were independently observed by transmission electron microscopy [22]. Fits to our heterogeneity model, plus a power law for the FM domains, are depicted by the solid lines in Fig. 2. We obtain $147 \pm 13 \AA$ for the average spacing between magnetic heterogeneities and $108 \pm 11 \AA$ for the variance in this spacing. The spheroidal form of the heterogeneities is prolate with semiprincipal axes $15 \pm 1 \AA$ and $51 \pm 4 \AA$. We find these fit parameters are field independent. Note, however, that the form of scatterers cannot be uniquely determined with SANS data. Scattering from an ellipsoid, for example, is indistinguishable to that from a distribution of polydisperse spheres [23].
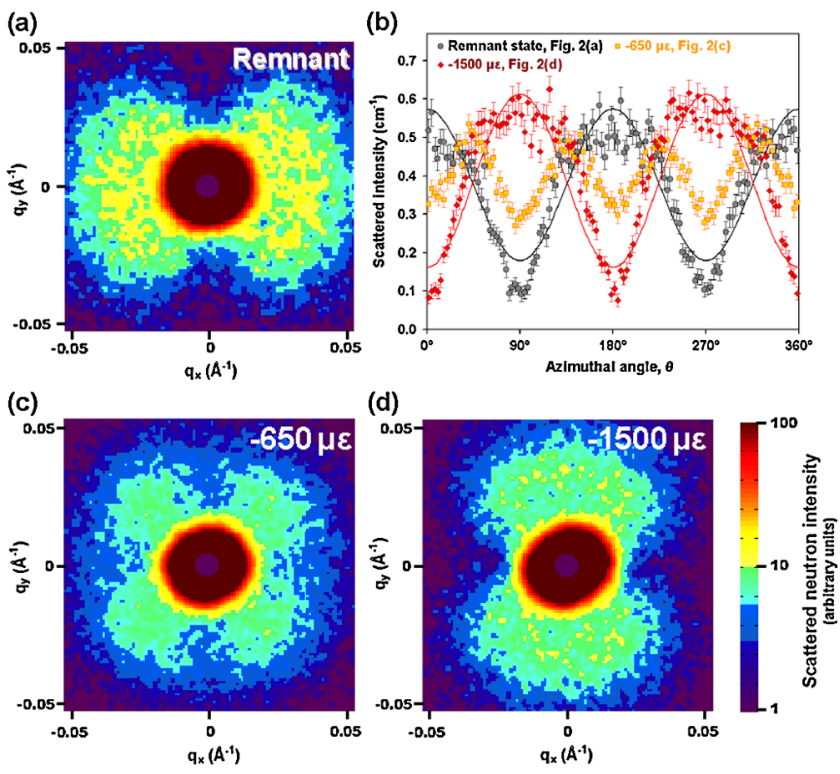

FIG. 3 (color online). Detector images using unpolarized neutrons with (a) neither field nor strain applied; compressive strains of (c) $-650 \mu \epsilon$, (d) $-1500 \mu \epsilon$ applied in the vertical direction $\hat{\mathbf{y}}$. (b) $\theta$ dependence, summed over $0.025<q<0.05 \AA^{-1}$. Solid lines are fits of the form $c_{1}+c_{2} \sin ^{2}(\theta-\phi)$.
In Fig. 3(a) we show the 2D scattering pattern before any field was applied. As anisotropy is observed [Fig. 3(b)], the heterogeneity magnetization must have a preferred direction: the sample has a remnant state, which possibly develops during sample growth. The orientation of the anisotropy shows this preferred direction is along $\hat{\mathbf{y}}$ [cf. Eq. (1a)]. Recalling the high field data [e.g., Fig. 1(c)], it is clear that applying a magnetic field reorients moments associated with the heterogeneities.

Figures 3(c) and 3(d) show the scattering under varying strain, with no magnetic field applied. Clearly heterogeneity moments also reorient under strain [Figs. 3(a) and 3(d)]. The strong scattering at low $q$ around the centers of each image indicates large scale FM domains, which persist even at high strains. Under intermediate strains, four lobes are observed [Fig. 3(c)]. These arise due to details in the form of $\mathbf{M}(\mathbf{R})$ around and within each heterogeneity. Four lobes with $90^{\circ}$ spacing in $\theta$ can be explained by a dipolar magnetic field surrounding the heterogeneity core [24]. Developing numerical methods such as micromagnetic simulation augur well for unraveling further details [25], and will be addressed in future work. We also observe four lobes at intermediate magnetic fields $\approx 10 \mathrm{mT}$, but these data are not shown here.

Under strain and magnetic field, FM domains proliferate and heterogeneity magnetization reorients, but how does each relate to the macroscopic magnetostriction? Figure 4 plots our results as functions of applied magnetic field and strain. In Fig. 4(a) we assess the reorientation of heterogeneity magnetization by examining horizontal $\hat{\mathbf{x}}$ and vertical $\hat{\mathbf{y}}$ sectors (summed $0.025<q<0.05 \AA^{-1}$ ) of the 2D pattern using unpolarized neutrons. We see that this reorientation occurs over the region $\approx 10 \mathrm{mT}$ [light (yellow) region]. Nearly all the magnetostriction is generated within a similar field range, as can be seen from the strain measured as a function of magnetic field. In contrast, the response of FM domains, characterized both by unpolarized scattering measured at low $q=0.005 \AA^{-1}$ and by the measured depolarization of initially polarized neutrons transmitted through the sample [16], is perceived over a higher field regime $\approx 60 \mathrm{mT}$ [darker (blue) region]. Considering, moreover, the responses observed under strain [Fig. 4(b)], it is clear the macroscopic magnetostriction and the magnetization reorienting in and around nanoscale heterogeneities are closely related. We suggest that heterogeneities may effect a localization of matrix magnetostriction, whereby distinct heterogeneity magnetizations create effective stray fields in the surrounding matrix, so engendering strain by coercing moments away from their easy directions, and bypassing the need to first shift FM domain walls at much larger scales. Differential x-ray absorption spectroscopy studies also suggest that magnetostrictive strain stems from the vicinity of heterogeneities [26]. We note, however, that localization is unable to account for the boost in $\lambda_{100}$ when samples are quenched [13]. Magnetostriction enhancement can be explained if 


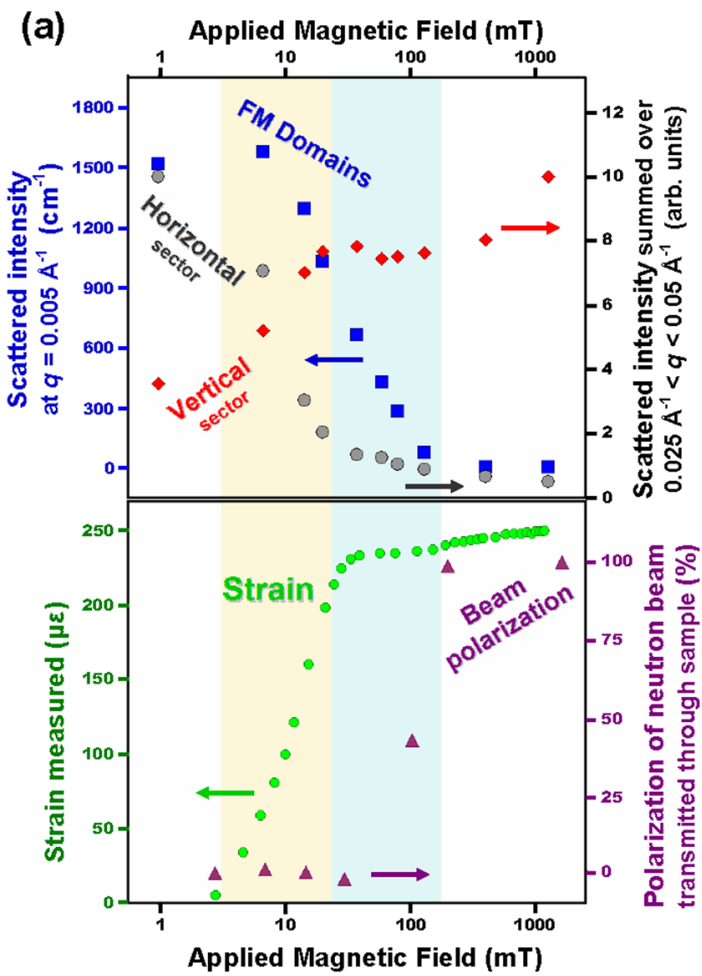

(b)

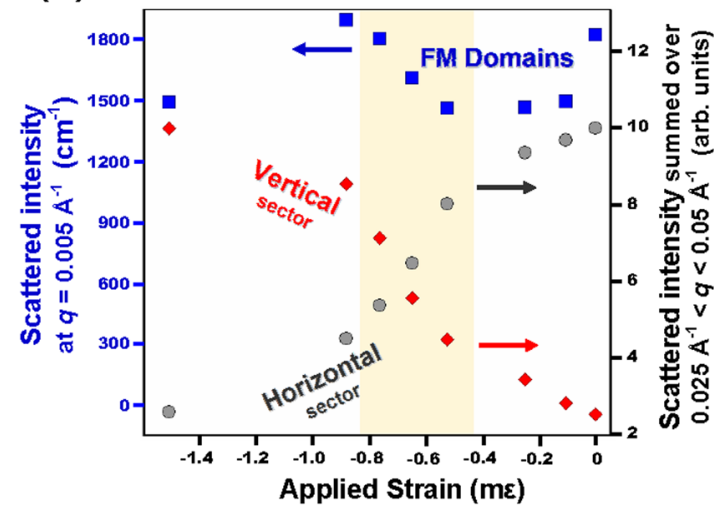

FIG. 4 (color online). Effect of applying (a) magnetic field or (b) compressive strain. The reorientation of heterogeneity magnetization is quantified by summing over horizontal (gray circles) and vertical (red diamonds) sectors on the 2D detector, and is highlighted by light (yellow) regions. The darker (blue) region highlights the FM domain response, characterized by the scattering at low $q=0.005 \AA^{-1}$ (blue squares) and by the depolarization of an initially polarized neutron beam (violet triangles). Green circles in (a) indicate the measured strain.

heterogeneities also generate strain. Indeed the idea of strain-generating heterogeneities, namely, of tetragonal $\mathrm{DO}_{22}$ minidomains proposed to form on cooling from $\mathrm{D}_{3}$ precipitates, has been put forward [7].

SANS is not sensitive in discriminating which heterogeneity phase is responsible for magnetostriction. Within our scattering model, negligible nuclear scattering would result-consistent with our data-from the ruck of conceivable phases, e.g., $\mathrm{D}_{22}, \mathrm{DO}_{3}$, ordered fcc $\mathrm{L}_{2}$, "B2- like" $\mathrm{L6} 6_{0}$, which all have nuclear SLDs very similar to the disordered A2 matrix. Meanwhile the inferred magnetic SLD contrast is $1.1 \times 10^{-6} \AA^{-2}$ - a difference of $\approx 0.5 \mu_{B}$ per $\mathrm{Fe}_{0.81} \mathrm{Ga}_{0.19}$ unit or $\approx 0.6 \mu_{B}$ per $\mathrm{Fe}$ atom. We note our simple model of monodisperse spheroids does not describe magnetization surrounding each heterogeneity which will affect these estimates. In conclusion, regardless of the details, magnetic nanoscale heterogeneities appear intertwined with $\mathrm{Fe}-\mathrm{Ga}$ magnetostriction.

We acknowledge financial support from ONR under MURI Grants No. N000140610530 and No. N000140110761, and from NSF under Grant No. DMR-0705368. Additional support is acknowledged by M. W. from ARO under MURI Grant No. 28D1083899, and by M.L. from DanScatt. This work utilized facilities supported in part by NSF under Agreement No. DMR0454672. We thank M. Al-Sheikhly for use of the irradiation facilities at the University of Maryland.

[1] E. Tatsumoto and T. Okamoto, J. Phys. Soc. Jpn. 14, 1588 (1959).

[2] G. Petculescu et al., J. Appl. Phys. 97, $10 \mathrm{M} 315$ (2005).

[3] R. A. Kellogg et al., Acta Mater. 52, 5043 (2004).

[4] P. D. McGary et al., J. Appl. Phys. 99, 08B310 (2006).

[5] T. Ueno et al., Sens. Actuators A, Phys. 148, 280 (2008).

[6] R. Wu, J. Appl. Phys. 91, 7358 (2002); J. Cullen, P. Zhao, and M. Wuttig, J. Appl. Phys. 101, 123922 (2007).

[7] A. G. Khachaturyan and D. Viehland, Metall. Mater. Trans. A 38, 2317 (2007).

[8] T. Khmelevska, S. Khmelevskyi, and P. Mohn, J. Appl. Phys. 103, 073911 (2008).

[9] E. W. Lee, Rep. Prog. Phys. 18, 184 (1955).

[10] T. A. Lograsso et al., J. Alloys Compd. 350, 95 (2003); Q. Xing et al., Acta Mater. 56, 4536 (2008).

[11] H. Cao et al., Phys. Rev. Lett. 102, 127201 (2009).

[12] For a recent review, see A. Michels and J. Weissmüller, Rep. Prog. Phys. 71, 066501 (2008).

[13] A. Clark et al., IEEE Trans. Magn. 37, 2678 (2001).

[14] R. M. Moon, T. Riste, and W. C. Koehler, Phys. Rev. 181, 920 (1969).

[15] P. A. Seeger and L. L. Daemen, Nucl. Instrum. Methods Phys. Res., Sect. A 457, 338 (2001), and references therein.

[16] O. Halpern and T. Holstein, Phys. Rev. 59, 960 (1941).

[17] W. Ruland, J. Appl. Crystallogr. 4, 70 (1971).

[18] C. Mudivarthi et al., J. Magn. Magn. Mater. 322, 2023 (2010).

[19] G. G. Long and L. E. Levine, Acta Crystallogr. Sect. A 61, 557 (2005).

[20] E. W. Huang et al., Appl. Phys. Lett. 93, 161904 (2008).

[21] F. Farsaci et al., Phys. Chem. Liq. 20, 205 (1989).

[22] M. Kumar and V. Vasudevan, Acta Mater. 44, 1591 (1996).

[23] P. Mittelbach and G. Porod, Acta Phys. Austriaca 15, 122 (1962).

[24] C. Vecchini et al., Appl. Phys. Lett. 87, 202509 (2005).

[25] S. Saranu et al., Phys. Status Solidi A 205, 1774 (2008).

[26] M. P. Ruffoni et al., Phys. Rev. Lett. 101, 147202 (2008). 